\title{
Parabolic Methods for the Construction of Spacelike Slices of Prescribed Mean Curvature in Cosmological Spacetimes
}

\author{
Klaus Ecker $^{1}$ and Gerhard Huisken ${ }^{2}$ \\ 1 Department of Mathematics, The University of Melbourne, Parkville, Vic. 3052, Australia \\ 2 Department of Mathematics, Faculty of Science, Australian National University, \\ GPO Box 4, Canberra, A.C.T. 2601, Australia
}

Received January 12, 1989

\begin{abstract}
Spacelike hypersurfaces of prescribed mean curvature in cosmological spacetimes are constructed as asymptotic limits of a geometric evolution equation. In particular, an alternative, constructive proof is given for the existence of maximal and constant mean curvature slices.
\end{abstract}

\section{Introduction}

In recent recent years spacelike hypersurfaces of prescribed mean curvature have played an important role in the study of Lorentzian manifolds. Maximal surfaces, i.e. surfaces of zero mean curvature were used in the first proof of the positive mass theorem [SY 1, SY 2] and in the analysis of the Cauchy problem for asymptotically flat spacetimes [CBY, LA], see also [MT] for further references. Spacelike hypersurfaces of constant mean curvature were studied in [CE, CY, TA]. In [GC] Gerhardt obtained general existence and regularity results for prescribed mean curvature surfaces in cosmological spacetimes and in [B 1] Bartnik settled the corresponding problem for asymptotically flat Lorentzian manifolds, see also [BS] for related results. In these articles the existence proofs are non-constructive as they rely on topological fixed point theorems.

In this paper we use parabolic equations to construct spacelike hypersurfaces of prescribed mean curvature in cosmological spacetimes $\mathscr{V}=\Sigma^{n} \times I$. In this setting a spacelike hypersurface can be written as the graph of a real valued function $u$ over some fixed Cauchy surface $\Sigma^{n}$ and the problem of finding a surface of prescribed mean curvature $\mathscr{H}$ reduces to a quasilinear elliptic equation for $u$. It was shown in $[\mathrm{GC}]$ that this equation can be solved under weak assumptions for $\mathscr{H}$ provided the existence of suitable barriers in the timelike future and past is known. In particular, it was proven that in cosmological spacetimes which satisfy the timelike convergence condition and admit a big bang and a big crunch there exists a foliation of $\mathscr{V}$ by hypersurfaces of constant mean curvature. For each $\mathscr{H} \equiv \tau \neq 0$ there is a unique surface $S_{\tau}$ of constant mean curvature $\tau$ and there exists at least 
one maximal hypersurface. If there are two different hypersurfaces of zero mean curvature $M_{1}$ and $M_{2}$, then the spacetime $\mathscr{V}$ has a product structure and is static between $M_{1}$ and $M_{2}$.

Here we study the following three basic problems:

(a) Given a function $\mathscr{H}$ on $\mathscr{V}$, construct a spacelike hypersurface with prescribed mean curvature $\mathscr{H}$; in particular, given $\tau$ construct the constant mean curvature surface $S_{\tau}$.

(b) Given a point $p_{0} \in \mathscr{V}$, construct the unique constant mean curvature surface passing through $p_{0}$.

(c) Given an arbitrary spacelike surface $M_{0}$, construct the unique constant mean curvature surface in $\mathscr{V}$ which bounds the same volume as $M_{0}$ (with respect to some fixed reference slice $\Sigma$ ).

In all three cases the required prescribed mean curvature surface will be obtained as the asymptotic limit of the solution to a suitable parabolic equation. We take an arbitrary initial surface $M_{0}$ and evolve it in the direction of its unit normal vector $v$ such that the speed at each point is determined by a difference $H-\mathscr{H}$, where $H$ is the mean curvature of the evolving surface and $\mathscr{H}$ is a forcing term depending on the particular problem. To be precise, let $M_{0}$ be given by an immersion

$$
\mathbf{F}_{0}: M^{n} \rightarrow \mathscr{V} \text {. }
$$

Then we solve the evolution equation

$$
\begin{gathered}
\frac{d}{d s} \mathbf{F}(p, s)=[(H-\mathscr{H}) \boldsymbol{v}](p, s) \\
\mathbf{F}(p, 0)=\mathbf{F}_{0}(p), \quad p \in M^{n}, \quad s \geqq 0 .
\end{gathered}
$$

where $\mathbf{v}(p, s)$ is the future directed unit normal to $M_{s}=\mathbf{F}(\cdot, s)\left(M^{n}\right)$ at $\mathbf{F}(p, s)$ and $H$ is the mean curvature on $M_{s}$.

The evolution of hypersurfaces by mean curvature, i.e. evolution Eq. (1) with $\mathscr{H} \equiv 0$ has been extensively studied in Riemannian ambient spaces, see $[\mathrm{H} 1, \mathrm{H} 2$, $\mathrm{H} 3, \mathrm{BK}]$. In the Riemannian case compact initial surfaces quickly contract and develop singularities as the flow decreases area. In the case of spacelike hypersurfaces in a Lorentzian manifold the mean curvature flow increases rather than decreases area which leads to a much more regular behaviour.

To solve problem (a) we choose $\mathscr{H}(p, s)=\mathscr{H}(\mathbf{F}(p, s))$ to be the prescribed mean curvature function on $\mathscr{V}$. We prove in Theorem 4.1 that under certain monotonicity conditions on $\mathscr{H}$ Eq. (1) has a smooth solution for all times provided $M_{s}$ remains in a smooth region of $\mathscr{V}$ and $\mathscr{V}$ satisfies the timelike convergence condition. If we assume the existence of barriers as in [GC] or [B 1] the surfaces $M_{s}$ will then asymptotically converge to a stationary solution of (1) as $s \rightarrow \infty$, see Theorem 4.2. In a subsequent paper we prove a corresponding result in the case of asymptotically flat spacetimes.

In Remark 4.10 we consider initial surfaces of strictly positive (say) mean curvature $H \geqq H_{0}>0$ and prove an a priori estimate for the proper time of curves $\gamma(s)=\mathbf{F}(p, s), p \in M^{n}$, where $\mathbf{F}(p, s)$ is a solution of (1) with $\mathscr{H} \equiv \tau>\max _{M_{0}} H$. It turns out that in this case the proper time of $\gamma$ is uniformly bounded for all such $\tau$ by $n / H_{0}$. This result is analogous to the well known fact that focal points occur on any past timelike geodesic emanating from $M_{0}$ of length exceeding $n / H_{0}$. 
To construct a constant mean curvature surface passing through a given point $x_{0} \in \mathscr{V}$, let $\mathbf{F}_{0}: M^{n} \rightarrow \mathscr{V}$ be an arbitrary spacelike initial surface with $\mathbf{F}_{0}\left(p_{0}\right)=x_{0}$ for some $p_{0} \in M^{n}$ and solve (1) with $\mathscr{H}(p, s)=H\left(p_{0}, s\right)$. The evolution equation then ensures that $M_{s}$ passes through $x_{0}$ at all times and we can again prove longtime existence of a smooth solution. Asymptotic convergence to a constant mean curvature surface can then be derived if we have an a priori estimate for the height of $M_{s}$, see Theorem 5.1. Such a height estimate is valid if barriers exist or if $\mathscr{V}$ satisfies a compactness condition which was first introduced by Geroch in [GR]. In [B 2] Bartnik solved the corresponding elliptic problem using the same compactness condition. Our approach to problem (b) only works in the Lorentzian case and fails in the corresponding situation in a Riemannian ambient space.

To obtain a constant mean curvature slice in $\mathscr{V}$ which encloses the same volume with respect to some fixed reference slice as a given initial surface $M_{0}$, we solve the initial value problem (1) with

$$
\mathscr{H}(s)=\int_{M_{s}} H(p, s) d \mu_{s} / \int_{M_{s}} d \mu_{s}
$$

being the mean value of the mean curvature on $M_{s}$. This flow was first used in [H 4] to deform convex hypersurfaces in Euclidean space into round spheres. The solution surfaces $M_{s}$ all bound the same volume as the initial surface $M_{0}$ and their area is increasing in the Lorentzian case. As in the first two models we prove longtime existence of a smooth solution and then obtain asymptotic convergence to a constant mean curvature surface provided $\mathscr{V}$ admits barriers or satisfies some compactness condition ensuring a height estimate for $M_{s}$, see Theorem 6.1.

To derive the existence and convergence results for Eq. (1) we have to establish a priori estimates for the height, the slope and the curvature of $M_{s}$. After fixing notation in Sect. 2 and calculating the relevant evolution equations in Sect. 3 we obtain these estimates in Sect. 4. The main results are then proved in Sects. 4-6.

\section{Preliminaries}

We consider $(n+1)$-dimensional smooth spacetimes $\mathscr{V}$ with a Lorentzian metric $\bar{g}=\left\{\bar{g}_{\alpha \beta}\right\}$ of signature $(-,+,+, \ldots,+)$. The metric pairing will be denoted by $\langle\cdot, \cdot\rangle$, the canonical connection by $\bar{\nabla}$ and the curvature tensor by $\bar{R} m=\left\{\bar{R}_{\alpha \beta \gamma \delta}\right\}$. Greek indices run from 0 to $n$. As in [B 1] we shall assume the existence of a global time function $t \in C^{\infty}(\mathscr{V})$ with nonzero, past-directed timelike vectorfield $\bar{\nabla} t$. The references slices $\mathscr{S}_{t}=\{p \in \mathscr{V} \mid t(p)=t\}$ have future-directed unit normal vector

$$
T=-\psi \bar{\nabla} t
$$

where the lapse function $\psi \in C^{\infty}(\mathscr{V})$ is defined by

$$
\psi^{-2}=-\langle\bar{\nabla} t, \bar{\nabla} t\rangle .
$$

We denote an adapted orthonormal frame for $\mathscr{S}_{t}$ by $\mathbf{e}_{0}, \mathbf{e}_{1}, \ldots, \mathbf{e}_{n}$ such that $\mathbf{e}_{0}=T$.

Now let $M^{n}$ be a smooth spacelike hypersurface in $\mathscr{V}$ which is embedded by some map

$$
\mathbf{F}: M^{n} \rightarrow \mathscr{V} .
$$

Let $\boldsymbol{v}$ be the future directed timelike unit normal and choose locally an adapted orthonormal frame $\tau_{0}, \tau_{1}, \ldots, \tau_{n}$ in $\mathscr{V}$ such that restricted to $M$ we have $\tau_{0}=\boldsymbol{v}$. We 
will denote by $g=\left\{g_{i j}\right\}$ and $R m=\left\{R_{i j k l}\right\}$ the induced metric and the Riemann curvature tensor on $M$ respectively, where Latin indices range from 1 to $n$. The second fundamental form $A=\left\{h_{i j}\right\}$ on $M$ is given by

$$
h_{i j}=\left\langle\bar{V}_{\tau_{i}} v, \tau_{j}\right\rangle=-\left\langle\bar{V}_{\tau_{i}} \tau_{j}, \boldsymbol{v}\right\rangle .
$$

As in $[\mathrm{H} 1]$ we write, summing over repeated indices

$$
\begin{gathered}
H=h_{i i}, \quad|A|^{2}=h_{i j} h_{i j}, \\
C=h_{i k} h_{k l} h_{l i} .
\end{gathered}
$$

The curvature, Ricci curvature Ric $=\left\{R_{i j}\right\}$ and scalar curvature $R$ on $M$ are given by Gauss' equation

$$
\begin{aligned}
R_{i j k l} & =\bar{R}_{i j k l}-h_{i k} h_{j l}+h_{i l} h_{j k}, \\
R_{i k} & =\bar{R}_{i k}-H h_{i k}+h_{i l} h_{l k}+\bar{R}_{i o k o}, \\
R & =\bar{R}-H^{2}+|A|^{2}+2 \overline{\operatorname{Ric}}(\boldsymbol{v}, \boldsymbol{v}) .
\end{aligned}
$$

The Codazzi equations state that

$$
\nabla_{k} h_{i j}-\nabla_{j} h_{i k}=\bar{R}_{o i j k}
$$

where $\nabla$ denotes covariant differentiation on $M$. Moreover, we have the following rule for the interchanging of derivatives:

$$
\nabla_{i} \nabla_{j} Y_{k}-\nabla_{j} \nabla_{i} Y_{k}=-R_{i j k l} Y_{l} .
$$

From these relations the following fundamental identities can be derived for the Laplacian of the second fundamental form, see e.g. [SSY] and [NS].

2.1. Lemma. We have the identities

(i) $\Delta h_{i j}=\nabla_{i} \nabla_{j} H+h_{i j}\left(|A|^{2}+\overline{\operatorname{Ric}}(\boldsymbol{v}, \boldsymbol{v})\right)-H h_{i k} h_{k j}$

$$
\begin{aligned}
& +2 h_{k l} \bar{R}_{l i j k}+h_{j l} \bar{R}_{l k i k}+h_{l i} \bar{R}_{l k j k}+H \bar{R}_{o i j o} \\
& +\bar{\nabla}_{k} \bar{R}_{o i j k}+\bar{\nabla}_{j} \bar{R}_{o k i k},
\end{aligned}
$$

(ii) $\frac{1}{2} \Delta|A|^{2}=|\nabla A|^{2}+h_{i j} \nabla_{i} \nabla_{j} H+|A|^{2}\left(|A|^{2}+\overline{\operatorname{Ric}}(\boldsymbol{v}, \boldsymbol{v})\right)-H C$

$$
\begin{aligned}
& +2 \bar{R}_{l i j k} h_{k l} h_{i j}+\bar{R}_{l k i k} h_{j l} h_{i j}+\bar{R}_{l k j k} h_{l i} h_{i j} \\
& +H \bar{R}_{o i j o} h_{i j}+\bar{V}_{k} \bar{R}_{o i j k} h_{i j}+\bar{V}_{j} \bar{R}_{o k i k} h_{i j} .
\end{aligned}
$$

In our a priori estimates it will be important to consider the height function $u$ given by

$$
u(p)=t(\mathbf{F}(p))
$$

and the gradient function $v$ which measures the angle between $M$ and the reference slicing $\mathscr{S}_{t}$

$$
v=-\langle\boldsymbol{v}, T\rangle .
$$

From $[\mathrm{B} 1,2.8]$ we quote the identity

$$
\Delta u=H \psi^{-1} v+\operatorname{div} \bar{\nabla} t,
$$

where $\Delta$ denotes the Laplace-Beltrami operator on $M$. Furthermore notice that

$$
\left\langle\tau_{\alpha}, \mathbf{e}_{\beta}\right\rangle \leqq v
$$


for all $0 \leqq \alpha, \beta \leqq n$ such that the restriction of any $p$-tensor $B \in T^{p}(\mathscr{V})$ to $M$ can be estimated by

$$
\left\|\left.B\right|_{T M}\right\| \leqq v^{p}\|B\| .
$$

For the convenience of the reader let us recall the first variation formula for $H$ (cf. [CB, FM, B 1]) with respect to a deformation of the surrounding space generated by an arbitrary transverse vector field $X$ :

$$
X\left(H_{X}\right)=-\Delta\langle X, \boldsymbol{v}\rangle+\langle X, \boldsymbol{v}\rangle\left(|A|^{2}+\overline{\operatorname{Ric}}(\boldsymbol{v}, \boldsymbol{v})\right)+\langle X, \nabla H\rangle .
$$

\section{Evolution Equations}

Starting from the evolution equation

$$
\frac{d}{d s} \mathbf{F}=(H-\mathscr{H}) \boldsymbol{v}
$$

we will derive evolution equations for the height, the gradient, the curvature and other relevant quantities on $M_{s}$.

Proceeding exactly as in [H 1] we first compute the derivative w.r.t.s of the metric $g$, volume element $\mu$ and the unit normal $v$ on $M$ :

3.1. Proposition. We have the equations

$$
\begin{aligned}
\frac{d}{d s} g_{i j} & =2(H-\mathscr{H}) h_{i j}, \\
\frac{d}{d s} \boldsymbol{v} & =\nabla(H-\mathscr{H}) \\
\frac{d}{d s} \mu & =H(H-\mathscr{H}) \mu .
\end{aligned}
$$

Now notice that

and

$$
\frac{d}{d s} u=(H-\mathscr{H}) \psi^{-1} v
$$

$$
\frac{d}{d s} v=-\langle T, \nabla(H-\mathscr{H})\rangle-(H-\mathscr{H})\left\langle\nabla_{v} T, \boldsymbol{v}\right\rangle,
$$

such that from (2) and (4) we obtain

3.2. Proposition. The height function and the gradient function satisfy

(i) $\left(\frac{d}{d s}-\Delta\right) u=-\mathscr{H} \psi^{-1} v-\operatorname{div} \bar{\nabla} t$,

(ii) $\left(\frac{d}{d s}-\Delta\right) v=-v\left(|A|^{2}+\overline{\operatorname{Ric}}(\boldsymbol{v}, \boldsymbol{v})\right)-T\left(H_{T}\right)+\langle T, \nabla \mathscr{H}\rangle-(H-\mathscr{H})\left\langle\nabla_{\mathbf{v}} T, \boldsymbol{v}\right\rangle$.

Finally we derive the following evolution equations for the second fundamental form. 
3.3. Proposition. We have the equations

$$
\begin{aligned}
\left(\frac{d}{d s}-\Delta\right) & h_{i j}=-\nabla_{i} \nabla_{j} \mathscr{H}-\mathscr{H}\left(h_{i k} h_{k j}+\bar{R}_{i o o j}\right) \\
& +2 H h_{i k} h_{k j}-h_{i j}\left(|A|^{2}+\overline{\operatorname{Ric}}(v, v)\right) \\
& -2 h_{k l} \bar{R}_{l i j k}-h_{j l} \bar{R}_{l k i k}-h_{l i} \bar{R}_{l k j k} \\
& \quad-\bar{\nabla}_{k} \bar{R}_{l i j k}-\bar{\nabla}_{j} \bar{R}_{o k i k}, \\
\frac{d}{d s} H= & \Delta(H-\mathscr{H})-(H-\mathscr{H})\left(|A|^{2}+\overline{\operatorname{Ric}}(v, v)\right),
\end{aligned}
$$

and the estimates

$$
\begin{aligned}
& \left(\frac{d}{d s}-\Delta\right)|A|^{2} \leqq-2|\nabla A|^{2}-|A|^{4}+c_{0}\left(1+|A|^{2}+\left|\nabla^{2} \mathscr{H}\right|^{2}\right), \\
& \left(\frac{d}{d s}-\Delta\right)\left|\nabla^{m} A\right|^{2} \leqq-2\left|\nabla^{m+1} A\right|^{2}+c_{m}\left(1+\left|\nabla^{m} A\right|^{2}+\left|\nabla^{m+2} \mathscr{H}\right|^{2}\right),
\end{aligned}
$$

where $m \geqq 0$ and

$$
c_{m}=c_{m}\left(m, n, v, \sum_{j=0}^{m+1}\left|\bar{\nabla}^{j} \bar{R}_{m}\right|, \sum_{j=0}^{m}\left|\bar{\nabla}^{j} \mathscr{H}\right|, \sum_{j=1}^{m}\left|\nabla^{j-1} A\right|\right) .
$$

Proof. Similarly as in [H 2] we derive

$$
\frac{d}{d s} h_{i j}=\nabla_{i} \nabla_{j}(H-\mathscr{H})+(H-\mathscr{H})\left(h_{i k} h_{k j}+\bar{R}_{\text {iooj }}\right)
$$

which implies (i) in view of Lemma 2.1 (i). The second identity is an immediate consequence of (4) and can also be derived by taking the trace of (i). Now observe that

$$
\frac{d}{d s}|A|^{2}=2 h_{i j} \frac{d}{d s} h_{i j}-4(H-\mathscr{H}) h_{i j} h_{j l} h_{l i}
$$

and the first inequality follows from (i), geometric-arithmetric mean inequalities and (3). As the different sign arising from the Lorentzian setting is irrelevant for proving the last inequality, we may proceed similarly as in [H 2], using again (3).

\section{Prescribing Mean Curvature}

In this and the following sections we assume that $\mathscr{V}$ is a cosmological spacetime, i.e. it is connected, globally hyperbolic and admits a compact Cauchy surface. This implies in particular the existence of a global time function $t \in C^{\infty}(\mathscr{V})$ as in Sect. 2. Moreover, we will always assume that the timelike convergence condition is satisfied, i.e.

$$
\overline{\operatorname{Ric}}(X, X) \geqq 0
$$

holds for all timelike vector fields $X$.

We are going to study the flow problem (1) with general $\mathscr{H} \in C^{\infty}(\mathscr{V})$ satisfying the monotonicity condition

$$
\langle\bar{\nabla} \mathscr{H}, X\rangle \geqq 0
$$


for all future directed timelike vectors $X$ and begin with the following long time existence result.

4.1. Theorem. Let $\mathscr{V}$ and $\mathscr{H}$ be as above and suppose that $M_{0}=\mathbf{F}_{0}\left(M^{n}\right)$ is a smooth compact spacelike hypersurface in $\mathscr{V}$. Then there exists a unique family $M_{s}=\mathbf{F}(\cdot, s)\left(M^{n}\right)$ of smooth compact spacelike hypersurfaces satisfying the initial value problem (1) on an interval $0 \leqq s<s_{0}$. Moreover, if $M_{s}$ remains in a smooth, compact region of $\mathscr{V}$ as $s \rightarrow s_{0}$, the solution can be extended beyond $s_{0}$.

In order to obtain asymptotic convergence to a stationary limiting hypersurface we have to impose additional conditions on $\mathscr{V}$. We say that two compact spacelike $C^{2}$-hypersurfaces $M^{ \pm}$are barrier surfaces for $M_{0}$ with respect to $\mathscr{H}$ if

and

$$
M^{ \pm} \subset I^{ \pm}\left(M_{0}\right)
$$

$$
\begin{array}{ll}
H_{M^{+}}(y)<\mathscr{H}(y) \quad \forall y \in M^{+}, \\
H_{M^{-}}(y)>\mathscr{H}(y) \quad \forall y \in M^{-} .
\end{array}
$$

Here $I^{ \pm}\left(M_{0}\right)$ denotes the future and past of $M_{0}$ respectively. Notice that for functions $\mathscr{H}$ which are globally bounded on $\mathscr{V}$ the existence of such barrier surfaces is guaranteed for arbitrary $M_{0}$ if we assume the occurrence of a "big bang" and a "big crunch," see [Ge] and [ES]. In this case one postulates the existence of times $T^{-}, T^{+}$with $-\infty<T^{-}<T^{+}<\infty$ and of sequences of compact spacelike slices $M_{k}^{ \pm}$satisfying

$$
\begin{gathered}
\left.\sup t\right|_{M_{\vec{k}}} \rightarrow T^{-}, \\
\left.\inf t\right|_{M_{k}^{+}} \rightarrow T^{+}
\end{gathered}
$$

such that $H_{M_{\bar{k}}} \rightarrow H_{-}$and $H_{M_{\vec{k}}^{+}} \rightarrow H_{+}$, where $-\infty \leqq H_{+}<H_{-} \leqq \infty$.

4.2. Theorem. Let $\mathscr{V}$ and $\mathscr{H}$ be as in Theorem 4.1 and assume in addition that two barrier surfaces $M^{ \pm}$exist for the initial hypersurface $M_{0}$ with respect to $\mathscr{H}$. Then the solution $M_{s}$ of (1) exists for all times $s>0$ and every sequence $\left(s_{k}\right) \rightarrow \infty$ has a subsequence $\left(s_{k^{\prime}}\right) \rightarrow \infty$ such that $M_{s_{k^{\prime}}}$, converges uniformly in $C^{\infty}$ to a smooth spacelike limiting hypersurface $M_{\infty}$ satisfying

$$
H_{M_{\infty}}=\left.\mathscr{H}\right|_{M_{\infty}} .
$$

We obtain asymptotic convergence to a unique limit in each of the following cases:

i) $\mathscr{V}$ satisfies the "ubiquitous energy condition" $\overline{\operatorname{R}} \operatorname{ic}(X, X)>0$ for all timelike vectors $X$.

ii) Some limiting surface $M_{\infty}$ has strictly positive or strictly negative mean curvature.

iii) $\mathscr{H} \equiv 0$.

4.3. Remark. (i) In cases i) and ii) the convergence occurs at an exponential rate. In case iii) there are two possibilities: Either $\mathscr{V}$ admits only one maximal slice in which case we get the estimate

$$
H_{\max }^{2}(s) \leqq \frac{n}{2} \cdot s^{-1},
$$

or there are at least two different maximal slices in $\mathscr{V}$. In the latter case there will be a whole layer of totally geodesic maximal slices, see [GC]. If the limiting slice is a 
boundary slice of this layer, $H(s)$ will decay as in (6). If the limiting slice is in the interior of the layer, the convergence will occur at an exponential rate due to the fact that the metric $\bar{g}$ of $\mathscr{V}$ is static inside the layer.

(ii) In a forthcoming paper we establish similar results under less restrictive assumptions on $\mathscr{H}$ and $\overline{\text { Ric. }}$

The crucial step in the proof of Theorem 4.1 is the gradient estimate.

4.4. Proposition. Let $M_{s}$ be a smooth solution of (1) on the interval $0 \leqq s<s_{0}$ such that $M_{s}$ is contained in a smooth compact subset $K \subset \mathscr{V}$ for all $0 \leqq s<s_{0}$. Then the gradient function $v$ satisfies the a priori estimate

where

$$
v(p, s) \leqq\left(1+\sup _{M_{0}} v\right) \sup _{(q, s) \in M^{n} \times\left[0, s_{0}\right]} \exp (\lambda[u(q, s)-u(p, s)]),
$$

$$
\lambda=\lambda\left(n, \psi,\|\mathscr{H}\|_{1, K},\|\bar{R} m\|_{0, K}, \sup _{M^{n} \times\left[0, s_{0}\right]}|H|\right) .
$$

Proof. Following the argument in [B 1, Theorem 3.1] let $\lambda>0$ be a constant to be chosen later and define

$$
C_{\lambda}=\left(1+\sup _{M_{0}} v\right) \sup _{M^{n} \times\left[0, s_{0}\right]} e^{\lambda u} .
$$

Suppose $e^{\lambda u} v$ reaches $C_{\lambda}$ for the first time at $\left(p_{1}, s_{1}\right) \in M^{n} \times\left(0, s_{0}\right]$. Then at this point

$$
\begin{aligned}
& 0 \leqq\left(\frac{d}{d s}-\Delta\right) e^{\lambda u} v, \\
& 0=\nabla\left(e^{\lambda u} v\right) .
\end{aligned}
$$

From Proposition 3.2 and [B 1, Proof of Theorem 3.1] we infer

$$
\left(\frac{d}{d s}-\Delta\right) u \leqq C_{0} v^{2}
$$

as well as

$$
\left(\frac{d}{d s}-\Delta\right) v \leqq-v|A|^{2}+C_{1}\left(v^{3}+v^{2}|A|\right),
$$

where

$$
C_{0}=C_{0}\left(\|\psi\|_{1, K},\|\mathscr{H}\|_{0, K}\right) \quad \text { and } \quad C_{1}=C_{1}\left(n,\|\psi\|_{1, K},\|\mathscr{H}\|_{1, K},\|\bar{R} m\|_{0, K}\right) .
$$

Here we also used the fact that $|\langle T, \nabla \mathscr{H}\rangle| \leqq v^{2}|\bar{\nabla} \mathscr{H}|$. Hence we conclude

$$
v|A|^{2} \leqq \lambda^{2} v|\nabla u|^{2}+\left(C_{0} \lambda+C_{1}\right) v^{3}+C_{1} v^{2}|A| .
$$

Proceeding as in [B 1, Theorem 3.1] we obtain

$$
v|A|^{2} \geqq\left(1+\frac{1}{2 n}\right) \lambda^{2} v|\nabla u|^{2}-C(n) v^{3}-H^{2} v .
$$

Since $|\nabla u|^{2}=\psi^{-2}\left(v^{2}-1\right)$ we finally arrive at

$$
\lambda^{2} \psi^{-2}\left(v^{2}-1\right) \leqq C_{2}(\lambda+1) v^{2}
$$

at $\left(p_{1}, s_{1}\right)$ where $C_{2}$ depends on $C_{0}, C_{1}, n$, and $H$. Since by definition of $C_{\lambda}$ we have $v\left(p_{1}, s_{1}\right) \geqq 2$, we derive a contradiction for large enough $\lambda$. Repeating the argument with $u$ replaced by $-u$ yields the result. 
To estimate the curvature on $M_{s}$ we need a technical lemma.

4.5. Lemma. Let $f$ be a function on $M^{n} \times\left[0, s_{0}\right]$ satisfying

$$
\left(\frac{d}{d s}-\Delta\right) f \leqq-a^{2} f^{2}+b^{2}
$$

for some constants $a, b \in \mathbb{R}$. Then we have

everywhere on $M^{n} \times\left[0, s_{0}\right]$.

$$
f \leqq \frac{b}{a}+\frac{1}{a^{2} s}
$$

Proof. Consider the function $g=s f-b s / a$. Then

$$
\left(\frac{d}{d s}-\Delta\right) g \leqq-2 a b g+g s^{-1}\left(1-a^{2} g\right)
$$

and we obtain a contradiction if $g$ reaches for the first time a value larger than $a^{-2}$. This proves the result.

4.6. Proposition. The mean curvature of $M_{s}$ satisfies

$$
\sup _{M^{n} \times\left[0, s_{0}\right]}|H| \leqq C\left(1+s^{-1}\right),
$$

where $C=C\left(n,\|\mathscr{H}\|_{0, K}\right)$.

Proof. Since $\mathscr{H}$ does not explicitly depend on $s$ here, we obtain from Proposition 3.3 (ii) and (1)

$$
\left(\frac{d}{d s}-\Delta\right)(H-\mathscr{H})=-(H-\mathscr{H})\left(|A|^{2}+\overline{\operatorname{Ric}}(\boldsymbol{v}, \boldsymbol{v})\right)+\langle\bar{\nabla} \mathscr{H}, \boldsymbol{v}\rangle .
$$

Since $|A|^{2} \geqq \frac{1}{n} H^{2}$ we estimate in view of (3)

$$
\left(\frac{d}{d s}-\Delta\right)(H-\mathscr{H})^{2} \leqq-2|\nabla(H-\mathscr{H})|^{2}-\frac{1}{n}(H-\mathscr{H})^{4}+C,
$$

where $C=\left(n,\|\mathscr{H}\|_{0, K}\right)$. Here we also need the timelike convergence condition and the monotonicity of $\mathscr{H}$. The estimate then follows from Lemma 4.5.

We can now prove estimates for the full second fundamental form and its higher derivatives.

4.7. Proposition. We have the estimate

$$
\sup _{M^{n} \times\left[0, s_{0}\right]}|A|^{2} \leqq C,
$$

where $C=C\left(n,\|v\|_{0, K},\|\bar{R} m\|_{1, K}, \sup _{M^{n} \times\left[0, s_{0}\right]}|H|,\|\mathscr{H}\|_{2, K}\right)$. Having bounded $v$ and $H$ we then obtain for general $m \geqq 1$,

$$
\sup _{M^{n} \times\left[0, s_{0}\right]}\left|\nabla^{m} A\right|^{2} \leqq C_{m}
$$

where $C_{m}=C_{m}\left(n, m,\|\bar{R} m\|_{m+1, K},\|\mathscr{H}\|_{m+2, K}, \sup _{M_{0}}\left|\nabla^{m} A\right|^{2}\right)$. 
Proof. We use the formula

$$
\nabla_{i} \nabla_{j} \mathscr{H}=\left\langle\tau_{i}, \mathbf{e}_{\alpha}\right\rangle\left\langle\tau_{j}, \mathbf{e}_{\beta}\right\rangle \bar{\nabla}_{\alpha} \bar{\nabla}_{\beta} \mathscr{H}+h_{i j}\langle\bar{\nabla} \mathscr{H}, \boldsymbol{v}\rangle
$$

to estimate

$$
\left|\nabla^{2} \mathscr{H}\right| \leqq v^{2}\left|\bar{\nabla}^{2} \mathscr{H}\right|+v|A||\bar{\nabla} \mathscr{H}|
$$

Hence we infer from Proposition 3.3 (iii)

$$
\begin{aligned}
\left(\frac{d}{d s}-\Delta\right)|A|^{2} & \leqq-|A|^{4}+C\left(1+|A|^{2}\right) \\
& \leqq-\frac{1}{2}|A|^{4}+C
\end{aligned}
$$

with $C=C\left(n,\|v\|_{0, K},\|\bar{R} m\|_{1, K}\|\mathscr{H}\|_{2, K}, \sup _{M^{n} \times\left[0, s_{0}\right]}|H|\right)$ and the conclusion follows from Lemma 4.5 .

In order to bound the higher derivatives of $A$ we estimate similarly as above

$$
\left|\nabla^{m+2} \mathscr{H}\right|^{2} \leqq c\left(m, v,\|\mathscr{H}\|_{m+2, K}, \sum_{j=0}^{m-1}\left|\nabla^{j} A\right|\right) \cdot\left(1+\left|\nabla^{m} A\right|^{2}\right) .
$$

Proposition 3.3 (iv) then yields

$$
\left(\frac{d}{d s}-\Delta\right)\left|\nabla^{m} A\right|^{2} \leqq-2\left|\nabla^{m+1} A\right|^{2}+C_{m}\left(1+\left|\nabla^{m} A\right|^{2}\right)
$$

where now $C_{m}=C_{m}\left(m, n,\|v\|_{0, K},\|\bar{R} m\|_{m+1, K},\|\mathscr{H}\|_{m+2, K}, \sum_{j=0}^{m-1}\left|\nabla^{j} A\right|\right)$. From here we can proceed by induction on $m$ as in [H4].

Proof of Theorem 4.1. If the initial surface $M_{0}$ is smooth, shorttime existence of a unique smooth solution of (1) follows from standard parabolic theory exactly as for the mean curvature flow in Riemannian manifolds. Since by assumption $M_{s}$ stays inside a compact smooth region of $\mathscr{V}, u$ is uniformly bounded. Hence we infer from Propositions 4.4, 4.6, 4.7 that $M_{s}$ converges to a smooth surface $M_{s_{0}}$ as $s \rightarrow s_{0}$. Thus the solution $M_{s}$ can be extended beyond $s_{0}$ by means of the local existence result.

4.8. Remark. i) The parabolic system (1) is equivalent to a single quasilinear parabolic equation for the height of $M_{s}$ over some fixed reference slice. This equation is uniformly parabolic provided $v$ is uniformly bounded. Hence it is possible to apply general parabolic theory to obtain longtime existence once the gradient estimate in Proposition 4.4 and the estimate for the mean curvature in Proposition 4.6 have been established, see [FA]. The geometric estimates in Proposition 4.7 however seem to be the more natural approach.

ii) As our curvature estimates in Proposition 4.7 are independent of the initial data, it is possible to obtain local existence assuming only Lipschitz initial data.

To establish Theorem 4.2 we begin with an a priori estimate for the height function.

4.9. Proposition. If $M^{ \pm}$are two barrier surfaces for $M_{0}$ with respect to $\mathscr{H}$, then the surfaces $M_{s}$ lie entirely in the interior of the compact region $K=K\left(M^{+}, M^{-}\right) \subset \mathscr{V}$ bounded by $\mathrm{M}^{+}$and $\mathrm{M}^{-}$for all $s>0$. 
Proof. By [B 1, Proposition 3.2] we may assume w.l.o.g. that the time function $t$ has been chosen such that $M_{0}=\{x \in \mathscr{V} \mid t(x)=0\}$ and $M^{ \pm}=\left\{x \in \mathscr{V} \mid t(x)=t^{ \pm}\right)$. We then want to prove

$$
t^{-}<u(p, s)<t^{+} \quad \forall s \geqq 0 \forall p \in M^{n} .
$$

Let us only prove the estimate from above. Suppose to the contrary that there exists a point $\left(p_{0}, s_{0}\right) \in M^{n} \times[0, \infty)\left(s_{0}>0\right)$, where $M_{s}$ touches $M^{+}$for the first time. At this point we have

$$
\frac{d}{d s} u \geqq 0 \text {. }
$$

From Proposition 3.2 (i) we conclude since $v$ and $\psi$ are non-negative by definition that

$$
H\left(p_{0}, s_{0}\right) \geqq \mathscr{H}\left(\mathbf{F}\left(p_{0}, s_{0}\right)\right),
$$

and hence in view of the barrier assumption

$$
H\left(p_{0}, s_{0}\right)>H_{M^{+}}\left(\mathbf{F}\left(p_{0}, s_{0}\right)\right) \text {. }
$$

Since the normal vectors of $M_{s_{0}}$ and $M^{+}$agree at this point we arrive at a contradiction to the strong elliptic maximum principle, [PW].

Having established the uniform height estimate we know from Theorem 4.1 that our solution $M_{s}$ exists for all times $s>0$ and it remains to prove asymptotic convergence to a stationary limit surface as in Theorem 4.2 . We will show that $(H-\mathscr{H})(s)$ converges to zero as $s \rightarrow \infty$. To this end let $\mathscr{V}_{s}$ be the volume enclosed by $M_{s}$ and some reference surface, e.g. $M^{-}$. Then we obtain from (1), Proposition 3.1 (iii) and the divergence theorem that

$$
\frac{d}{d s}\left(\left|M_{s}\right|+\int_{\mathscr{V}_{s}} \mathscr{H} d V\right)=\int_{M_{s}}(H-\mathscr{H})^{2} d \mu_{s} .
$$

Since $\left|M_{s}\right|$ and $\left|\mathscr{V}_{s}\right|$ are uniformly bounded in view of our height estimate in Proposition 4.9 we conclude that

$$
\int_{0}^{\infty} \int_{M_{s}}(H-\mathscr{H})^{2} d \mu_{s} d s<\infty .
$$

Moreover, from our uniform estimates for all curvature quantities and their derivatives in Proposition 4.7 we infer as in [H 4] that both

$$
\int_{M_{s}}(H-\mathscr{H})^{2} d \mu_{s} \text { and } \frac{d}{d s} \int_{M_{s}}(H-\mathscr{H})^{2} d \mu_{s}
$$

are uniformly bounded in $s$. Thus $\int_{\mathcal{M}_{s}}(H-\mathscr{H})^{2} d \mu_{s}$ tends to zero as $s \rightarrow \infty$. The uniform estimate for the derivative of $(H-\mathscr{H})$ in conjunction with a standard interpolation argument then shows that

$$
\sup _{M_{s}}|H-\mathscr{H}| \underset{s \rightarrow \infty}{\longrightarrow} 0 \text {. }
$$

Thus every sequence $M_{s_{k}}$ has a subsequence converging to some stationary asymptotic limit, proving the first convergence result in Theorem 4.2.

We now turn to studying additional assumptions which guarantee the convergence of $M_{s}$ to a unique stationary limit: 
(i) If $\operatorname{Ric}(X, X)>0$ for all timelike vector fields $X$ then in view of the estimates in Proposition 4.4 and Proposition 4.9 there is $\delta=\delta\left(M^{ \pm}\right)>0$ such that

$$
\overline{\operatorname{Ric}}(\boldsymbol{v}, \boldsymbol{v}) \geqq \delta .
$$

Furthermore, from the monotonicity assumption on $\mathscr{H}$ we have

$$
\langle\bar{\nabla} \mathscr{H}, \boldsymbol{v}\rangle \geqq 0
$$

and hence obtain in view of Proposition 3.3 (ii)

$$
\left(\frac{d}{d s}-\Delta\right)(H-\mathscr{H})^{2} \leqq-2 \delta(H-\mathscr{H})^{2},
$$

which immediately implies

$$
\sup _{M_{s}}(H-\mathscr{H})^{2} \geqq e^{-2 \delta s} \sup _{M_{0}}(H-\mathscr{H})^{2} .
$$

This clearly yields exponential convergence to a smooth stationary limit surface $M_{\infty}$ in view of Proposition 3.2 (i).

(ii) Suppose $H_{M_{\infty}}>0$ for some limiting surface $M_{\infty}=\mathbf{F}_{\infty}\left(M^{n}\right), \mathbf{F}_{\infty}=\lim _{s_{k} \rightarrow \infty} \mathbf{F}_{s_{k}}$. Since

$$
\lim _{s \rightarrow \infty} \sup _{M_{s}}|H-\mathscr{H}|=0
$$

and $\mathscr{H} \circ \mathbf{F}_{\infty}>0$ we have for $s \geqq s_{0}$ sufficiently large

$$
H(s)>\delta\left(s_{0}\right)>0 .
$$

This yields $|A|^{2} \geqq \frac{1}{n} \delta^{2}$ and we can use the timelike convergence condition and the monotonicity condition on $\mathscr{H}$ in conjunction with Proposition 3.3 (ii) to obtain similarly as in (i),

$$
\left(\frac{d}{d s}-\Delta\right)(H-\mathscr{H})^{2} \leqq-\frac{2}{n} \delta^{2}(H-\mathscr{H})^{2},
$$

again implying the desired exponential convergence to $M_{\infty}$.

(iii) Suppose that $\mathscr{H} \equiv 0$. Then Proposition 3.3 (ii) implies in view of $|A|^{2} \geqq \frac{1}{n} H^{2}$ that

$$
\left(\frac{d}{d s}-\Delta\right) H^{2} \leqq-\frac{2}{n} H^{4},
$$

which by Lemma 4.5 yields inequality (6).

In order to prove uniqueness of the asymptotic limit we have to distinguish two cases. If $\mathscr{V}$ admits only one maximal slice we are done. If $\mathscr{V}$ contains at least two different maximal slices we infer from [GC] that they are both totally geodesic and that all maximal surfaces form a layer of totally geodesic surfaces. More precisely, we may assume w.1.o.g. ([B 1, Proposition 3.2]) that the references slices

$$
\mathscr{S}_{t}=\{X \in \mathscr{V} \mid t(x)=t\}
$$

form a layer $\mathscr{C}_{0}=\bigcup_{t_{0} \leqq t \leqq t_{1}} \mathscr{S}_{t}$ of totally geodesic maximal slices, where $-\infty \leqq t_{0}$ $<t_{1} \leqq \infty$. Inside $\mathscr{C}_{0}{ }_{0} \leqq t \leqq t_{1}$ the metric $\bar{g}$ of $\mathscr{V}$ splits isometrically into

$$
\bar{g}=-d t^{2}+g_{\mathscr{S}_{t_{0}}}
$$


Since in particular $\bar{\nabla} t$ is a constant vector in $\mathscr{C}_{0}$, the evolution equation for the height function $u$ in Proposition 3.2 (i) reduces to

inside this region.

$$
\left(\frac{d}{d s}-\Delta\right) u=0
$$

Suppose first that for some sequence of times $s_{k} \rightarrow \infty M_{s_{k}}$ converges to a totally geodesic maximal slice $\mathscr{S}_{t}$ in the interior of $\mathscr{C}_{0}$, i.e. $t_{0}<t<t_{1}$. Then by the parabolic maximum principle we have that $M_{s}$ is in the interior of $\mathscr{C}_{0}$ for all $s \geqq s_{k_{0}}$ sufficiently large. We then compute from (7) and Proposition 3.1 (iii),

$$
\frac{d}{d s} \int_{M_{s}}(u-\bar{u})^{2} d \mu_{s} \leqq-2 \int_{M_{s}}|\nabla u|^{2} d \mu_{s}+\int_{M_{s}} H^{2}(u-\bar{u})^{2} d \mu_{s}
$$

for $s \geqq s_{k_{0}}$, where $\bar{u}(s)=\left|M_{s}\right|^{-1} \int_{M_{s}} u d \mu_{s}$. In view of our uniform bounds for the geometry of $M_{s}$ the Poincaré inequality

$$
\int_{M_{s}}(u-\bar{u})^{2} d \mu_{s} \leqq c_{0} \int_{M_{s}}|\nabla u|^{2} d \mu_{s}
$$

holds with some fixed constant $c_{0}$ independently of $s$. Moreover, we see from (6) that $\sup H^{2} \leqq \varepsilon$ for $s \geqq s_{\varepsilon} \geqq s_{k_{0}}$ and therefore conclude for $\varepsilon \leqq c_{0}^{-1}$

$$
\frac{d}{d s} \int_{M_{s}}(u-\bar{u})^{2} d \mu_{s} \leqq-c_{0}^{-1} \int_{M_{s}}(u-\bar{u})^{2} d \mu_{s} .
$$

Together with our uniform estimates and standard interpolation inequalities this implies exponential decay of $\sup _{M_{s}}|u-\bar{u}|$ and exponential convergence of $M_{s}$ to $\mathscr{S}_{t}=M_{\infty}$.

Suppose now there exists a sequence $s_{k} \rightarrow \infty$ such that $M_{s_{k}}$ converges to $M_{\infty}=\mathscr{S}_{t_{0}}\left(\right.$ or $\left.M_{\infty}=\mathscr{S}_{t_{1}}\right)$. Then for arbitrary $\varepsilon>0$ we can find $k_{\varepsilon}$ such that $u\left(s_{k_{\varepsilon}}\right)$ $\leqq t_{0}+\varepsilon$. Since $\mathscr{S}_{t_{0}+\varepsilon}$ is maximal and therefore a stationary solution of the flow it acts as a barrier to $M_{s}$ and the strong parabolic maximum principle yields

$$
u(s) \leqq t_{0}+\varepsilon
$$

for every $s \geqq s_{k_{\varepsilon}}$, proving that $M_{s}$ converges to the unique limit $\mathscr{S}_{t_{0}}=M_{\infty}$. Without further assumptions we don't expect faster convergence than in (6) in this case.

4.10. Remark. i) Let $M_{0}$ be a compact Cauchy surface in $\mathscr{V}$ such that inf $H \geqq H_{0}>0$. Let $\mathbf{F}_{s}^{\sigma}$ be the solution of (1) with $\mathscr{H} \equiv \sigma \geqq \max H$ and define the

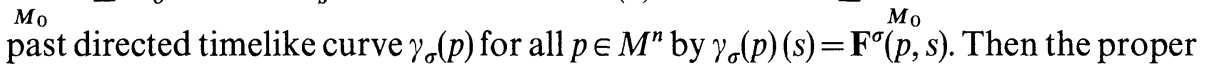
length of $\gamma_{\sigma}(p)$ can be estimated by $n / H_{0}$ independently of $\sigma$ and $p$. This follows from an analysis of the inequality

$$
\frac{d}{d s} H_{\min } \geqq-\frac{1}{n} H_{\min }^{2}\left(H_{\min }-\sigma\right)
$$

obtained from Proposition 3.3 (ii).

ii) It would be very interesting to find reasonable conditions for $M_{0}$ and $\mathscr{V}$ which imply that the solution of (1) with $\mathscr{H} \equiv 0$ converges to a maximal surface, without having to assume the existence of barriers. 


\section{Constant Mean Curvature Cauchy Surfaces Passing Through a Given Point}

In this section let $\mathscr{V}$ be a cosmological spacetime satisfying the timelike convergence condition and let $y_{0} \in \mathscr{V}$ be fixed. Given a compact Cauchy surface $M_{0}=\mathbf{F}_{0}\left(M^{n}\right)$ with $\mathbf{F}_{0}\left(p_{0}\right)=y_{0}$ for some $p_{0} \in M^{n}$ we want to construct a unique constant mean curvature surface $M_{\infty}$ passing through $y_{0}$ by solving evolution equation (1) with $\mathscr{H} \equiv \mathscr{H}(s)=H\left(p_{0}, s\right)$. We show that this approach succeeds provided $\mathscr{V}$ satisfies a suitable compactness condition.

5.1. Theorem. Let the spacetime $\mathscr{V}$ and the Cauchy surface $M_{0}$ be given as above. Assume furthermore either of the following conditions holds:

(a) $\mathscr{V} \sim I\left(y_{0}\right)$ is compact.

(b) There exist two smooth compact spacelike barrier surfaces $M^{ \pm} C I^{ \pm}\left(M_{0}\right)$ satisfying

$$
H_{M^{+}}<\inf _{M_{0}} H \leqq \sup _{M_{0}} H<H_{M^{-}} .
$$

Then problem (1) with $\mathscr{H}(s)=H\left(p_{0}, s\right)$ has a unique smooth solution for all $s>0$ which converges to a unique constant mean curvature surface passing through the point $y_{0}$.

5.2. Remark. (i) It was shown in [B 2] that condition (a), introduced in [GR], implies the existence of a constant mean curvature surface passing through $y_{0}$.

(ii) If we assume the "ubiquitous energy condition" $\overline{\operatorname{Ric}}(X, X)>0$ for all timelike vectors $X$, or if the mean curvature of the limiting surface is nonzero, then the convergence in Theorem 6.1 occurs at an exponential rate.

Proof of Theorem 5.1. As a first step we show that either of the conditions a) or b) implies an a priori height bound for the surfaces $M_{s}:$ From the definition of the flow problem (1) with $\mathscr{H}(s)=H\left(p_{0}, s\right)=H_{p_{0}}$ we know that all surfaces $M_{s}$ contain $y_{0}$. Since the $M_{s}$ are spacelike condition (a) implies that they have to stay inside the fixed compact region $\mathscr{V} \sim I\left(y_{0}\right)$ during the whole flow. In case of condition (b) we first observe that

$$
\sup _{M_{s}}|H| \leqq \sup _{M_{0}}|H| .
$$

This is an immediate consequence of Proposition 3.3 (ii), the timelike convergence condition and the parabolic maximum principle. From here we conclude as in Proposition 4.9 that $M^{+}$and $M^{-}$act as barriers for the surfaces $M_{s}$, proving the desired height bound. Since all other a priori estimates of Sect. 4 carry over, we have existence of a longtime solution and it remains to establish asymptotic convergence. From [MT, GC, Theorem 7.3] we know that Cauchy surfaces with nonzero constant mean curvature in $\mathscr{V}$ are unique. Moreover there cannot be two distinct maximal slices passing through $y_{0}$. Hence it suffices to show that

$$
\lim _{s \rightarrow \infty}\left|\sup _{M_{s}} H-\inf _{M_{s}} H\right|=0 .
$$

Using Proposition 3.3 (ii), the timelike convergence condition and $|A|^{2} \geqq \frac{1}{n} H^{2}$ we infer for the Lipschitz continuous functions $H_{\max }(s)=\sup _{M_{s}} H$ and $H_{\min }(s)=\inf _{M_{s}} H$ 
the inequalities

$$
\begin{aligned}
\frac{d}{d s} H_{\max } & \leqq-\frac{1}{n}\left(H_{\max }-H_{p_{0}}\right) H_{\max }^{2}, \\
\frac{d}{d s} H_{\min } & \geqq-\frac{1}{n}\left(H_{\min }-H_{p_{0}}\right) H_{\min }^{2},
\end{aligned}
$$

and hence

$$
\frac{d}{d s}\left(H_{\max }-H_{\min }\right) \leqq \frac{1}{n}\left(\left(H_{\max }-H_{p_{0}}\right) H_{\max }^{2}-\left(H_{\min }-H_{p_{0}}\right) H_{\min }^{2}\right) .
$$

(i) Suppose there exists $s_{0} \geqq 0$ such that

$$
H_{\max }\left(s_{0}\right)<0 \text { or } H_{\min }\left(s_{0}\right)>0 \text {. }
$$

In view of (8) and (9) we then have

$$
H_{\max }(s)<-\varepsilon \text { or } H_{\min }(s)>\varepsilon
$$

for all $s \geqq s_{0}$ and some $\varepsilon>0$. This however implies by (10)

$$
\frac{d}{d s}\left(H_{\max }-H_{\min }\right) \leqq-\frac{\varepsilon^{2}}{n}\left(H_{\max }-H_{\min }\right)
$$

such that $\left(H_{\max }-H_{\min }\right)$ converges to zero exponentially fast in this case.

(ii) Assume that for all $s \geqq 0$ we have

$$
H_{\max }(s) \geqq 0 \quad \text { and } \quad H_{\min }(s) \leqq 0 .
$$

If there are sequences $s_{k}^{+} \rightarrow \infty$ and $s_{k}^{-} \rightarrow \infty$ such that

$$
H_{\max }\left(s_{k}^{+}\right) \rightarrow 0 \text { and } H_{\min }\left(s_{k}^{-}\right) \rightarrow 0
$$

we conclude again from (8) and (9) that $\lim _{s \rightarrow \infty} H_{\max }(s)=\lim _{s \rightarrow \infty} H_{\min }(s)=0$. Suppose this is not true. Then we may assume w.l.o.g. that for some fixed $\delta>0 H_{\max }(s) \geqq \delta$ for all $s>0$. In fact, by (8) we may assume

$$
\lim _{s \rightarrow \infty} H_{\max }(s)=\delta>0 .
$$

Moreover, suppose there exists a sequence $s_{k} \rightarrow \infty$ such that

$$
\left(H_{\max }-H_{p_{0}}\right)\left(s_{k}\right) \geqq \beta>0
$$

for some $\beta>0$. In view of Proposition 4.7 we have uniform estimates for $H, H_{p_{0}}$ and all their derivatives, so we may find a positive constant $\alpha=\alpha(\beta)>0$ independent of $s_{k}$ such that

$$
H_{\max }\left(s_{k}\right)-H_{p_{0}}\left(s_{k}\right) \geqq \frac{\beta}{2}
$$

uniformly in each interval $\left[s_{k}, s_{k}+\alpha(\beta)\right]$. This yields in view of (8)

$$
\frac{d}{d s} H_{\max } \leqq-\frac{1}{n} \frac{\beta}{2} \delta^{2}
$$


in these intervals. This however forces $H_{\max }$ to become negative in finite time contradicting (12). Hence it remains only to consider the case where in addition to (11) and (12) we have

$$
\lim _{s \rightarrow \infty}\left(H_{\max }(s)-H_{p_{0}}(s)\right)=0 .
$$

Now let $p_{s} \in M^{n}$ be such that $H\left(p_{s}, s\right)=H_{\text {min }}(s)$. In view of (11) and our uniform estimates for $|\nabla H|$ we can find for arbitrary $\varepsilon>0$ a radius $\varrho_{\varepsilon}$ such that

$$
H(p, s) \leqq \varepsilon \quad \forall p \in B_{\varrho_{\varepsilon}}\left(p_{s}\right),
$$

where $B_{\varrho_{\varepsilon}}\left(p_{s}\right)$ is a geodesic ball around $p_{s}$ satisfying $\left|B_{\varrho_{\varepsilon}}\left(p_{s}\right)\right| \geqq c_{\varepsilon}>0$ independently of $s$. For the volume $\mathscr{V}_{s} \subset \mathscr{V}$ enclosed by $M_{s}$ and some fixed reference slice, we have the identity

$$
\frac{d}{d t}\left|\mathscr{V}_{s}\right|=-\int_{M_{s}}\left(H-H_{p_{0}}\right) d \mu_{s} .
$$

In view of our uniform height estimate there exists a sequence $s_{l} \rightarrow \infty$ such that

Furthermore we can write

$$
\int_{M_{s_{l}}}\left(H-H_{p_{0}}\right)\left(s_{l}\right) d \mu_{s_{l}} \rightarrow 0 .
$$

$$
\int_{M_{s}}\left(H-H_{p_{0}}(s)\right) d \mu_{s}=\int_{M_{s} \backslash B_{Q_{\varepsilon}}\left(p_{s}\right)}\left(H-H_{p_{0}}(s)\right) d \mu_{s}+\int_{B_{Q_{\varepsilon}}\left(p_{s}\right)}\left(H-H_{p_{0}}(s)\right) d \mu_{s} .
$$

By (13) and the uniform estimate on $\left|M_{s}\right|$ the first integral can be made arbitrarily small whereas in view of (12), (13), (14) and the inequality $\left|B_{\varrho_{\varepsilon}}\left(p_{s}\right)\right| \geqq c_{\varepsilon}$ the second integral can be estimated by

$$
\begin{aligned}
\int_{\boldsymbol{B}_{\varepsilon}\left(p_{s}\right)}\left(H-H_{p_{0}}(s)\right) d \mu_{s} & \leqq\left|B_{\varrho_{\varepsilon}}\left(p_{s}\right)\right|\left(\varepsilon-\frac{\delta}{2}\right) \\
& \leqq c_{\varepsilon}\left(\varepsilon-\frac{\delta}{2}\right) \leqq-c_{\varepsilon} \frac{\delta}{4}<0
\end{aligned}
$$

if $s$ is sufficiently large and $\varepsilon<\frac{\delta}{4}$. But this contradicts (15). Hence in case (ii) we must also have $\lim _{s \rightarrow \infty} H_{\max }(s)=\lim _{s \rightarrow \infty} H_{\min }(s)=0$.

In case we assume $\overline{\operatorname{R}} \stackrel{s \rightarrow \infty}{i c}(X, X)>0$ for all timelike vectors $X$ we actually have

$$
\overline{\operatorname{Ric}}(v, v) \geqq c_{0}>0
$$

in view of our height and gradient estimates. Hence Proposition 3.3 (ii) yields

$$
\frac{d}{d s}\left(H_{\max }-H_{\min }\right) \leqq-c_{0}\left(H_{\max }-H_{\min }\right)
$$

which immediately implies an exponential rate of convergence.

\section{Constant Mean Curvature Cauchy Surfaces Enclosing a Prescribed Volume}

In this section we consider the volume preserving flow given by problem (1) with $\mathscr{H} \equiv \mathscr{H}(s)=H(s)$, where

$$
\bar{H}(s)=\left|M_{s}\right|^{-1} \int_{M_{s}} H d \mu_{s}
$$


is the average of the mean curvature on $M_{s}$. This flow was studied in [H 4] for the Riemannian case.

6.1. Theorem. Let $\mathscr{V}$ be a cosmological spacetime satisfying the timelike convergence condition. Let $M_{0} \subset \mathscr{V}$ be a smooth compact Cauchy surface enclosing a volume $\mathscr{W}$ with respect to some fixed reference slice $\Sigma$. Assume furthermore that $\mathscr{V}$ satisfies one of the following compactness conditions:

a) There exist two smooth compact spacelike barrier surfaces $M^{ \pm} \subset I^{ \pm}\left(M_{0}\right)$ satisfying

$$
H_{M^{+}}<\inf H_{M_{0}} \leqq \sup H_{M_{0}}<H_{M^{-}} .
$$

b) For each $y \in M_{0}, \mathscr{V} \sim I\left(y_{0}\right)$ is compact.

Then there exists a unique longtime solution $M_{s}=\mathbf{F}_{s}\left(M^{n}\right)$ of problem (1) with $\mathscr{H}(s)$ $=\bar{H}(s)$ which converges exponentially fast in $C^{\infty}$ to a smooth compact constant mean curvature Cauchy surface $M_{\infty}$ enclosing the same volume with $\Sigma$ as $M_{0}$.

\subsection{Remark. Condition b) was used in [GG].}

Proof of Theorem 6.1. From Proposition 3.3 (ii) with $\mathscr{H}=\bar{H}$ and the timelike convergence condition we conclude that

$$
\frac{d}{d s} \sup _{M_{s}} H \leqq 0 \text { and } \quad \frac{d}{d s} \inf _{M_{s}} H \geqq 0 .
$$

Thus, if condition a) is satisfied, we obtain a height-bound as in Proposition 4.9. In case of condition b) the height estimate follows from the fact that $M_{s}$ intersects $M_{0}$ for all $s$, since it encloses the same volume with respect to $\Sigma$ as $M_{0}$. All the other estimates of Sect. 4 carry over unchanged since in this case

$$
\sup _{M_{s}}|\mathscr{H}| \leqq \sup _{M_{0}}|H|
$$

and $\nabla \mathscr{H} \equiv 0$.

This establishes the existence of a smooth solution of the volume preserving flow for all $s>0$. It remains only to prove the asymptotic convergence. We proceed as in Sect. 4, following [H 4] to prove

$$
\sup |H-\bar{H}| \rightarrow 0 \quad \text { as } s \rightarrow \infty \text {. }
$$

In fact, as $\mathscr{H}$ is the mean value of $H$, we even have

$$
\frac{d}{d s}\left|M_{s}\right|=\int_{M_{s}}(H-\bar{H})^{2} d \mu_{s}
$$

in view of Proposition 3.1 (iii). We now compute using Proposition 3.1 (iii) and Proposition 3.3 (ii)

$$
\frac{d}{d s} \int_{M_{s}}(H-\bar{H})^{2} d \mu_{s} \leqq-2 \int_{M_{s}}|\nabla H|^{2} d \mu_{s}+\int_{M_{s}}|H|(H-\bar{H})^{3} d \mu_{s} .
$$

By (16) and (17) we actually have

$$
\frac{d}{d s} \int_{M_{s}}(H-\bar{H})^{2} d \mu_{s} \leqq-2 \int_{M_{s}}|\nabla H|^{2} d \mu_{s}+\varepsilon \int_{M_{s}}(H-\bar{H})^{2} d \mu_{s}
$$


for $s \geqq s_{\varepsilon}$. We now proceed as in the proof of Theorem 4.1. In view of our uniform a priori estimates for the geometry of $M_{s}$ we can apply the Poincaré inequality with a constant $c_{0}$ independent of $s$. If we choose $\varepsilon$ small enough we finally arrive at

$$
\frac{d}{d s} \int_{M_{s}}|H-\bar{H}|^{2} d \mu_{s} \leqq-c_{0}^{-1} \int_{M_{s}}|H-\bar{H}|^{2} d \mu_{s}
$$

which establishes the desired exponential convergence result for $\sup _{\boldsymbol{M}_{s}}|H-\bar{H}|$ in view of standard interpolation inequalities. This immediately yields exponential convergence of $M_{s}$ to a limiting surface $M_{\infty}$.

\section{References}

[B 1] Bartnik, R.: Existence of maximal surfaces in asymptotically flat spacetimes. Commun. Math. Phys. 94, 155-175 (1984)

[B 2] Bartnik, R.: Remarks on cosmological spacetimes and constant mean curvature surfaces. Commun. Math. Phys. 117, 615-624 (1988)

[BS] Bartnik, R., Simon, L.: Spacelike hypersurfaces with prescribed boundary values and mean curvature. Commun. Math. Phys. 87, 131-152 (1982)

[BK] Brakke, K.A.: The motion of a surface by its mean curvature. Math. Notes, Princeton, N.J.: Princeton University Press 1978

[BF] Brill, D., Flaherty, F.: Maximizing properties of extremal surfaces in general relativity. Ann. Inst. Henri Poincaré A 28, 335-347 (1978)

[BR] Budic, R. et al.: On the determination of Cauchy surfaces from intrinsic properties. Commun. Math. Phys. 61, 87-95 (1978)

[CE] Calabi, E.: Examples of Bernstein problems for some non-linear equations. AMS Symposium on global analysis, Berkeley 1968

[CY] Cheng, S.Y., Yau, S.-T.: Maximal space-like hypersurfaces in the Lorentz-Minkowski spaces. Ann. Math. 104, 407-419 (1976)

[CB] Choquet-Bruhat, Y.: Maximal submanifolds and submanifolds with constant mean extrinsic curvature of a Lorentzian manifold. Ann. Sci. Norm. Sup. Pisa, Sér. IV. 3, 361-376 (1976)

[CBY] Choquet-Bruhat, Y., York, J.: The Cauchy problem. General Relativity and Gravitation, Held, A. (ed.) New York: Plenum Press 1980

[ES] Eardley, D., Smarr, L.: Time functions in numerical relativity: Marginally bound dust collapse. Phys. Rev. D 19, 2239-2259 (1979)

[EH] Ecker, K., Huisken, G.: Mean curvature evolution of entire graphs. Ann. Math. 130, 453-471 (1989)

[FM] Fischer, A., Marsden, J.: Topics in the dynamics of General Relativity, in Isolated Gravitating systems in General Relativity (Italian Phys. Soc. 1979), 322-395

[FA] Friedman, A.: Partial differential equations of parabolic type. Englewood Cliffs, N.J.: Prentice-Hall 1964

[GG] Galloway, G.: Splitting theorems for spatially closed spacetimes. Commun. Math. Phys. 96, 423-429 (1984)

[GC] Gerhardt, C.: $H$-surfaces in Lorentzian manifolds. Commun. Math. Phys. 89, 523-553 (1983)

[GR] Geroch, R.: Singularities in closed universes. Phys. Rev. Lett. 17, 445-447 (1966)

[GA] Goddard, A.J.: Foliations of space-time by space-like hypersurfaces of constant mean curvature. Commun. Math. Phys. 54, 279-282 (1977)

[HE] Hawking, S., Ellis, G.: The large-scale structure of spacetime. Cambridge: Cambridge University Press 1973

[H 1] Huisken, G.: Flow by mean curvature of convex surfaces into spheres. J. Diff. Geometry 10, 237-266 (1984)

[H 2] Huisken, G.: Contracting convex hypersurfaces in Riemannian manifolds by their mean curvature. Invent. Math. 84, 463-480 (1986) 
[H 3] Huisken, G.: Deforming hypersurfaces of the sphere by their mean curvature. Math. Z. 195, 205-219 (1987)

[H 4] Huisken, G.: The volume preserving mean curvature flow. J. Reine Angew. Math. 382, 35-48 (1987)

[LA] Lichnerowicz, A.: L'intégration des équations de la gravitation relativiste e le problème des n corps. J. Maths. Pures Appl. 23, 37-63 (1944)

[MT] Marsden, J.E., Tipler, F.J.: Maximal hypersurfaces and foliations of constant mean curvature in general relativity. Phys. Rep. 66, (3) 109-139 (1980)

[NS] Nishikawa, S.: On maximal spacelike hypersurfaces in a Lorentzian manifold. Nagoya Math. J. 95, 117-124 (1984)

[PW] Protter, M.H., Weinberger, H.F.: Maximum principles in differential equations. Englewood Cliffs, N.J.: Prentice Hall 1967

[SSY] Schoen, R., Simon, L., Yau, S.-T.: Curvature estimates for minimal hypersurfaces. Acta Math. 134, 275-288 (1975)

[SY 1] Schoen, R., Yau, S.-T.: Proof of the positive mass theorem. I. Commun. Math. Phys. 65, 45-76 (1979)

[SY 2] Schoen, R., Yau, S.-T.: Proof of the positive mass theorem. II. Commun. Math. Phys. 79, 231-260 (1981)

[TA] Treibergs, A.E.: Entire space-like hypersurfaces of constant mean curvature in Minkowski space. Invent. Math. 66, 39-56 (1982)

Communicated by S.-T. Yau 
